\title{
MODELAGEM E SIMULAÇÃO DE UMA UNIDADE DE ADSORÇÃO PARA REMOÇÃO DE CONTAMINANTES DE CORRENTES DE REFINARIAS
}

\author{
E. Q. EUGENIO, A. L. H. COSTA e E. R. A. LIMA \\ Universidade do Estado do Rio de Janeiro, Programa de Pós Graduação em Engenharia Química \\ E-mail para contato: erikaqueiros@ hotmail.com.br
}

\begin{abstract}
RESUMO - A implantação de legislações restritivas, que visam à minimização dos teores de enxofre nos combustíveis, tem incentivado a comunidade acadêmica a pesquisar alternativas complementares aos processos usuais de hidrotratamento. Entre as opções investigadas, a adsorção tem se mostrado uma possibilidade promissora, porém há poucos estudos na literatura que investiguem a avaliação do sistema completo, abrangendo as operações de adsorção/dessorção. Desta forma, o presente trabalho busca simular a remoção de contaminantes de correntes de refinarias em um sistema de colunas operando em uma condição de estado estacionário cíclico. O modelo matemático proposto é composto por um sistema de equações diferenciais parciais referentes às equações de conservação. A resolução do problema é realizada através do método das linhas com discretização via diferenças finitas. A aplicação da rotina de simulação desenvolvida é ilustrada através de um exemplo típico de uma operação industrial de uma refinaria.
\end{abstract}

\section{INTRODUÇÃO}

De forma geral, os contaminantes sulfurados são a maior preocupação nas frações de petróleo, pois além de serem venenos e inibidores para catalisadores metálicos, estes são um fator de poluição ambiental via emissões de $\mathrm{SO}_{\mathrm{x}}$ durante a queima de combustíveis. Com o intuito minimizar o impacto desses componentes, a resolução $n^{\circ} 50$ da ANP de 2013 restringiu a comercialização do óleo diesel a um teor máximo de $10 \mathrm{mg} / \mathrm{kg}$.

A hidrodessulfurização (HDS) de frações de petróleo é o processo com maior utilização nas refinarias para a produção de tais combustíveis com baixo teor de enxofre. No entanto, a redução dos níveis de enxofre abaixo de $10 \mathrm{mg} / \mathrm{kg}$ dificilmente é alcançado por catalisadores convencionais nas condições de processo do HDS (WHITEHURST,1998). Dentre as alternativas complementares ao processo de refino, a adsorção se mostra uma operação promissora, devido à alta eficiência e economia energética, uma vez que não há consumo de hidrogênio e suas condições de temperatura e pressão são mais brandas em relação ao processo tradicional.

O processo de dessulfurização por adsorção baseia-se na capacidade seletiva de um material 
sólido (adsorvente) em remover, por meio de forças intermoleculares, compostos sulfurados (adsorbato) da corrente de hidrocarbonetos. O adsorvente normalmente é acondicionado em uma coluna de leito fixo. Já a regeneração do adsorvente é feita geralmente percolando o leito com um dessorvente em fluxo contracorrente, tendo por resultado uma corrente com elevada concentração dos compostos orgânicos de enxofre.

O presente trabalho objetiva simular a dinâmica de uma unidade de adsorção até atingir o estado estacionário cíclico e com fluxo contracorrente das cargas de adsorção e dessorção em uma coluna de leito fixo. O modelo investigado contempla o efeito da resistência à transferência de massa e dispersão axial no leito durante o preenchimento da coluna, e a descrição da concentração de equilíbrio do adsorbato é feita pela isoterma não linear de Langmuir-Freundlich. O método numérico utilizado para a resolução das equações diferenciais parciais foi o método das linhas via diferenças finitas centrais.

\section{SISTEMA INVESTIGADO}

O sistema proposto no estudo consiste em duas colunas de adsorção operando em etapas alternadas concomitantemente, no qual um ciclo do processo pode ser considerado como uma sequência de 4 etapas: adsorção, esgotamento da corrente de refinaria, dessorção e esgotamento do dessorvente, conforme demonstrado na figura 1.

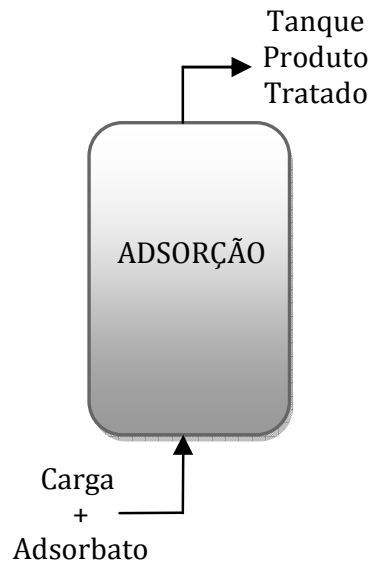

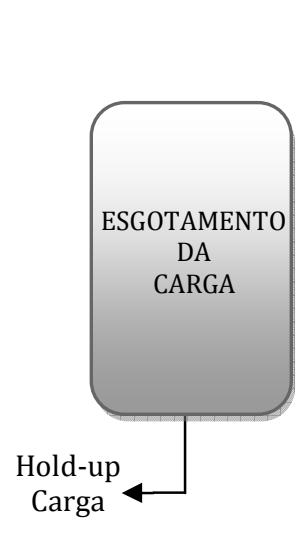

Figura 1: Esquematização do ciclo da unidade de adsorção

Na etapa de adsorção, a corrente de refinaria percorre o leito em um fluxo ascendente, cuja corrente de saída é encaminhada ao tanque de produto tratado. Como condição inicial no primeiro ciclo, o adsorvente se encontra virgem e o leito vazio de carga, por este motivo, o preenchimento do 


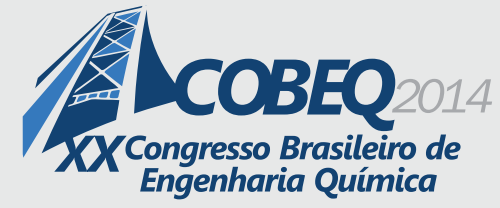

leito é simulado através da modulação do coeficiente de dispersão axial e coeficiente global de transferência de massa, dada a posição da frente líquida. Ao fim da primeira etapa, o volume remanescente da carga presente no leito é drenado instantaneamente e direcionado a um tanque com elevada concentração de adsorbato.

$\mathrm{Na}$ etapa de dessorção, o dessorvente é alimentado em fluxo descendente e seu efluente é encaminhado à unidade de recuperação. A simulação do preenchimento do leito é realizada de forma similar a etapa de adsorção. Por fim, após a dessorção, o solvente remanescente na fase fluida é instantaneamente descarregado e igualmente encaminhado para a unidade de recuperação.

\section{MODELAGEM MATEMÁTICA}

Na formulação das equações de conservação de massa dos processos de adsorção e dessorção, adotaram-se as seguintes hipóteses: (a) Partículas esféricas uniformes, (b) processo isotérmico, (c) fluxo unidimensional na direção axial, (d) perfil de velocidade uniforme, associada à dispersão do adsorbato e (e) resistência à transferência de massa no sistema descrita por um coeficiente global de transferência associado a uma força motriz linear.

Conforme as hipóteses assumidas, o principio de conservação de massa na fase fluida para as espécies químicas adsorvidas em volume de controle arbitrário é dado pela equação:

$$
\frac{\delta C_{i, j, k}}{\delta t}=\left(\frac{\delta D_{i, j, k}}{\delta z}-v_{z}\right) \cdot \frac{\delta C_{i, j, k}}{\delta z}+D_{i, j, k} \frac{\delta^{2} C_{i, j, k}}{\delta z^{2}}-\frac{(1-\varepsilon)}{\varepsilon} \cdot \rho_{s o l} \frac{\delta q_{i, j, k}}{\delta t}
$$

na qual, $C_{i, j, k}$ e $q_{i, j, k}$ representam as concentrações dos adsorbatos nas fases fluida e sólida, respectivamente, $\mathrm{D}_{\mathrm{i}, \mathrm{j}, \mathrm{k}}$ a dispersão axial, $\varepsilon$ a porosidade do leito, $\rho_{\text {sol }}$ a densidade aparente do leito, $v_{z}$ velocidade intersticial do fluido e os índices $i$ referem-se às espécies contaminantes, $j$ à etapa operacional de adsorção ou dessorção e $k$ ao ciclo em operação da unidade.

Já a equação de conservação na fase sólida é descrita por (RUTHVEN,1984):

$$
\frac{\partial q_{i, j, k}}{\partial t}=\frac{6 \cdot k_{i, j, k}}{d_{p}}\left(q_{e q, i, j, k}-q_{i, j, k}\right)
$$

em que, $k_{i}$ representa o coeficiente global de transferência de massa; a fração $6 / d_{p}$ equivale a área interfacial das partículas esféricas por unidade de volume, e $q_{e q, i}$ é a concentração de equilíbrio da fase sólida, descrita pela isoterma multicomponentes de adsorção de Langmuir-Freundlich:

$$
q_{e q, i, j, k}=\frac{q_{\max , j} K_{i, j, k} C_{i, j, k}^{m_{i, j, k}}}{1+\sum_{i=1}^{n c} K_{i, j, k} C_{i, j, k}^{m_{i, j, k}}}
$$




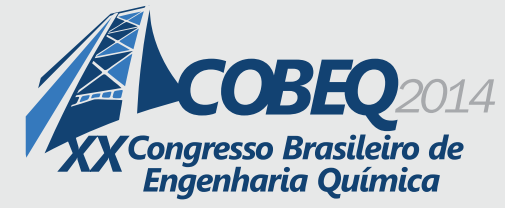

sendo, $K$ e $m$ os parâmetros da isoterma multicomponente de Langmuir-Freundlich e $q_{\max }$ a capacidade máxima de adsorção do adsorbato, que por sua vez é utilizado como parâmetro de adimensionamento da concentração dos contaminantes na fase sólida.

A fim de minimizar erros numéricos, foi realizada a metodologia de adimensionamento, sendo utilizados os parâmetros de referência: $T$ o tempo de residência, $L$ a altura coluna, $C_{i 0}$ concentração de contaminantes presentes na carga a ser adsorvida e $q_{\max , j}$, variável relacionada à concentração máxima adsorvida das operações de adsorção e dessorção.

Para resolução das equações de conservação (1) e (2), as etapas do processo de adsorção estão, individualmente, sujeitas às condições de contorno e inicial. Assim, no primeiro ciclo de adsorção, estas condições são:

$$
\begin{array}{lll}
\mathrm{t}=0 & (0<\mathrm{Z}<\mathrm{L}) & \overline{C_{l, 1,1}}=0 ; \overline{q_{l, 1,1}}=0 \\
\mathrm{z}=0 & (\mathrm{t}>0) & \left.v_{z} C_{i, 1, k}\right|_{z=0}-\left.D_{i, 1, k} \frac{\partial C_{i, 1, k}}{\partial z}\right|_{z=0}=v_{z} C_{i, 1, k_{-} 0} \\
\mathrm{z}=\mathrm{L} & (\mathrm{t}>0) & \left.\frac{\partial C_{i, 1, k}}{\partial z}\right|_{z=L}=0
\end{array}
$$

Nestas equações, o índice $j=1$ refere-se à etapa de adsorção, enquanto $j=2$ referir-se-á à etapa de dessorção, e $C_{i, j, k_{-} o}$ equivale à concentração de alimentação da etapa em operação da unidade de adsorção.

A etapa de esgotamento da carga é modelada pelo descarregamento imediato da fase fluida, de modo que a concentração da fase sólida se mantenha igual ao estágio final da etapa de adsorção.

$$
\mathrm{t}=\mathrm{t}_{\text {final }} \quad(0<\mathrm{z}<\mathrm{L}) \quad \overline{C_{l, 2, k}}=0 ; \quad \overline{q_{l, 2, k}}=\overline{q_{l, 1, k}}
$$

sendo esta a condição inicial da etapa de dessorção. Já as condições de contorno são evidenciadas pela inversão do ponto de referência em relação à etapa de adsorção. Assim, as condições de contorno são invertidas em relação às da etapa de adsorção:

$$
\begin{array}{ll}
\mathrm{z}=0 & \left.(\mathrm{t}>0) \quad \frac{\partial C_{i, 2, k}}{\partial z}\right|_{z=0}=0 \\
\mathrm{z}=\mathrm{L} & \left.(\mathrm{t}>0) \quad v_{z} C_{i, 2, k}\right|_{z=L}-\left.D_{i, 2, k} \frac{\partial C_{i, 2, k}}{\partial z}\right|_{z=L}=v_{z} C_{i, 2, k_{-} 0}
\end{array}
$$

Por fim, a etapa de esgotamento do dessorvente é análoga à etapa de esgotamento da carga, a qual conduz à condição inicial do próximo ciclo de adsorção.

$$
\mathrm{t}=\mathrm{t}_{\text {final }} \quad(0<\mathrm{z}<\mathrm{L}) \quad \overline{C_{l, 1, k}}=0 ; \quad \overline{q_{l, 1 . k}}=\overline{q_{l, 2, k-1}}
$$




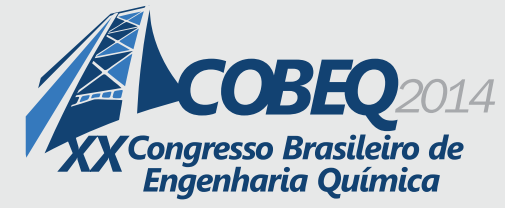

\section{PROCEDIMENTO NUMÉRICO}

A solução numérica do sistema de equações diferenciais parciais e suas condições iniciais e de contorno foi obtida utilizando o método das linhas por discretização no espaço via diferenças finitas no domínio do problema. O algoritmo computacional foi desenvolvido em linguagem Scilab utilizando a ferramenta DASSL (Differential Algebraic System Solver) para integração no tempo.

Como ferramenta para expressar o preenchimento do leito foi utilizada uma função de regularização para a propriedade de dispersão axial, garantindo a continuidade da propriedade e minimizando erros de aproximação do método numérico. A função de regularização empregada no trabalho ao longo do leito consiste na função tangente hiperbólica (FREITAS, 2012):

$D(\bar{z})=\left[1-\frac{1+\tanh \left(\frac{\bar{z}-z^{*}}{\eta}\right)}{2}\right] D_{i, j}$

sendo $z^{*}$ o ponto discretizado no espaço referente à interface da carga durante o preenchimento do leito e $\eta$ parâmetro de suavização da curva. No entanto, a modulação do coeficiente global de transferência de massa foi realizada através de uma função degrau de acordo com a posição da interface.

Foi adotada como condição de parada para a convergência do problema de estado estacionário cíclico a norma do vetor de concentração adimensional do adsorbato no adsorvente ao final de dois ciclos consecutivos da etapa de dessorção.

A partir da análise das curvas de ruptura, obtém-se a concentração do soluto em função do volume do efluente coletado $\left(V_{f}\right)$, a qual se refere à quantidade do soluto não retido pelo leito em um dado tempo de operação. Assim:

$C_{\text {média }}=\frac{\int_{0}^{t f}\left(C_{i, j}\right) \cdot Q \cdot d t}{Q\left(t_{f}-T\right)}=\frac{\int_{0}^{t f}\left(C_{i, j}\right) \cdot Q \cdot d t}{V_{f}}$

sendo $Q$ a vazão volumétrica.

\section{RESULTADOS}

Utilizando o algoritmo desenvolvido foi possível determinar perfis de concentração para qualquer intervalo de tempo, e em qualquer posição ao longo dos leitos da unidade. Desta forma, a partir dos dados típicos de dimensão de uma coluna de adsorção, propriedades de zeólitas (BASMADJIAN, 1997) e da isoterma de Langmuir-Freundlich (CERUTTI, 2007), expressos na Tabela 1, obtiveram-se os resultados de simulação apresentados na Figura 2. 
Tabela 1 - Dados de entrada e parâmetros do modelo adotados na simulação.

\begin{tabular}{|c|c|c|}
\hline Altura do Leito, L & \multicolumn{2}{|c|}{$5 \mathrm{~m}$} \\
\hline Diâmetro do Leito, D & \multicolumn{2}{|c|}{$3 \mathrm{~m}$} \\
\hline Diâmetro da partícula, $d_{p}$ & \multicolumn{2}{|c|}{$0,4 \mathrm{~cm}$} \\
\hline Densidade Aparente do Leito, $\rho_{\text {sol }}$ & \multicolumn{2}{|c|}{$1500 \mathrm{~g} / \mathrm{L}$} \\
\hline Porosidade do leito, $\varepsilon$ & \multicolumn{2}{|c|}{0,82} \\
\hline & \multicolumn{2}{|c|}{ Parâmetros do Programa } \\
\hline $\mathrm{N}^{\mathrm{o}}$ de Pontos de Discretização, $\mathrm{n}$ & \multicolumn{2}{|c|}{70} \\
\hline $\mathrm{N}^{\mathrm{o}}$ de ciclos de operação & \multicolumn{2}{|c|}{5} \\
\hline \multirow[t]{2}{*}{ Parâmetro de Suavização, $\eta$} & \multicolumn{2}{|c|}{0,005} \\
\hline & Adsorção & Dessorção \\
\hline Vazão de entrada, Q & $200 \mathrm{~m}^{3} / \mathrm{h}$ & $200 \mathrm{~m}^{3} / \mathrm{h}$ \\
\hline Concentração de Entrada, $\mathrm{C}^{0}$ & $3000 \mathrm{mg} / \mathrm{L}$ & $0 \mathrm{mg} / \mathrm{L}$ \\
\hline Massa específica da carga, $\rho_{\mathrm{L}}$ & 0,81 & 0,81 \\
\hline Tempo do Ciclo de Operação & $43,47 \mathrm{~min}$ & $43,47 \mathrm{~min}$ \\
\hline Coef. Dispersão Axial, $\mathrm{D}_{\mathrm{i}}$ & $3,2795 \mathrm{~cm}^{2} / \mathrm{min}$ & $3,2795 \mathrm{~cm}^{2} / \mathrm{min}$ \\
\hline \multirow[t]{2}{*}{ Coef. global transf. Massa, $\mathrm{k}$} & $0,0035 \mathrm{~cm} / \mathrm{min}$ & $0,0035 \mathrm{~cm} / \mathrm{min}$ \\
\hline & \multicolumn{2}{|c|}{ Isoterma de Langmuir-Freundlich } \\
\hline Capacidade Máx. de Adsorção, $\mathrm{q}_{\max }$ & $0,05154 \mathrm{~g} / \mathrm{g}$ & $0,005154 \mathrm{~g} / \mathrm{g}$ \\
\hline Constante de Equilíbrio de LF, K & $6 \times 10^{12}\left(\mathrm{~cm}^{3} / \mathrm{g}\right)^{3}$ & $6 \times 10^{12}\left(\mathrm{~cm}^{3} / \mathrm{g}\right)^{3}$ \\
\hline Constante de LF, $\mathrm{m}$ & 3 & 3 \\
\hline
\end{tabular}
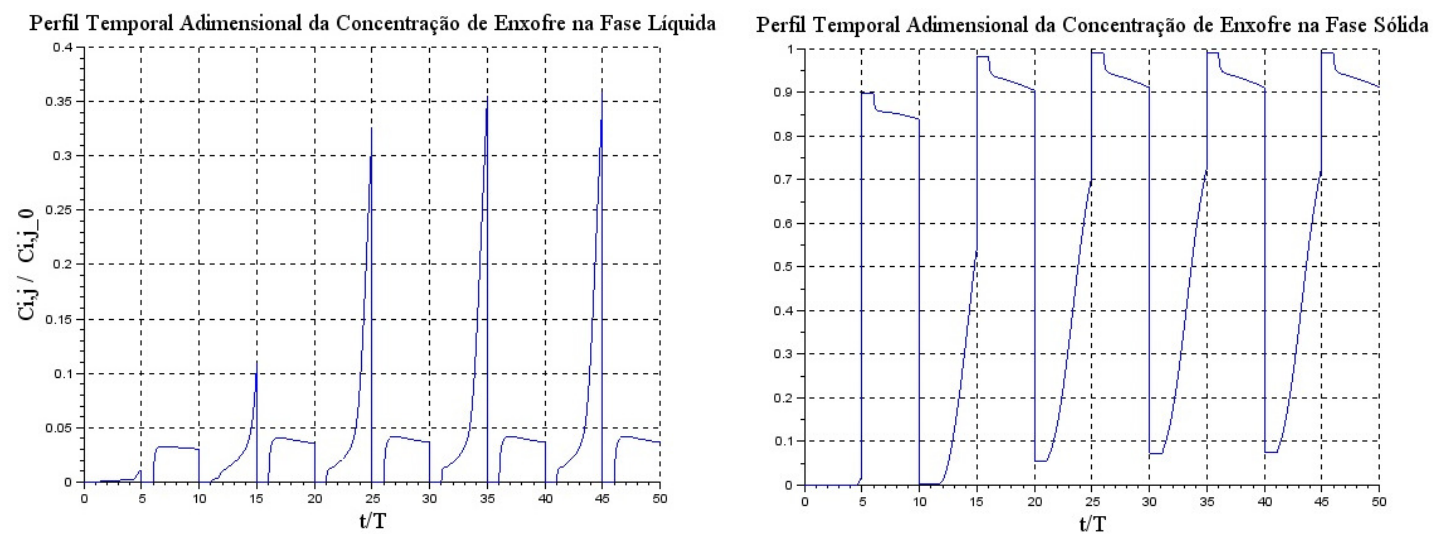

Figura 2 - Perfis temporais da concentração adimensional do adsorbato na fase líquida e na fase sólida 
A Figura 2 apresenta o perfil temporal adimensionado das curvas de ruptura das operações contínuas de adsorção e dessorção até alcançar o estado estacionário cíclico, bem como o perfil correspondente de concentração de adsorbato na fase sólida ao longo do tempo. Estes resultados indicam que o modelo matemático proposto descreve bem o comportamento da operação de adsorção e dessorção em uma coluna de leito fixo e que foram necessário 5 ciclos de operação para atingir o estado estacionário.

Foram realizados testes para verificar a influência do tamanho da malha computacional e do parâmetro de suavização da função de regularização da dispersão axial empregados para a solução numérica. Para o primeiro teste, foi constatada uma grande influência do número de subdivisões da malha sobre os resultados obtidos, de modo que a malha contendo 70 pontos de discretização foi adotada por apresentar praticamente o mesmo resultado de malhas mais refinadas, exigindo um menor esforço computacional e consequentemente menor tempo de processamento. Em relação ao parâmetro de suavização da função de regularização, foi constatado que não há variação significativa no impacto da dispersão axial para os valores de 0,005 e 0,01 . Deste modo, o valor 0,005 foi adotado por ser o menor valor possível no parâmetro de suavização sem haver danos à solução numérica.

Na Figura 3 e na Tabela 2, são apresentados os resultados referentes às concentrações e volumes do eluente, da carga remanescente na etapa de esgotamento e do dessorvente coletado nas etapas de dessorção e esgotamento após alcançar o estado estacionário:
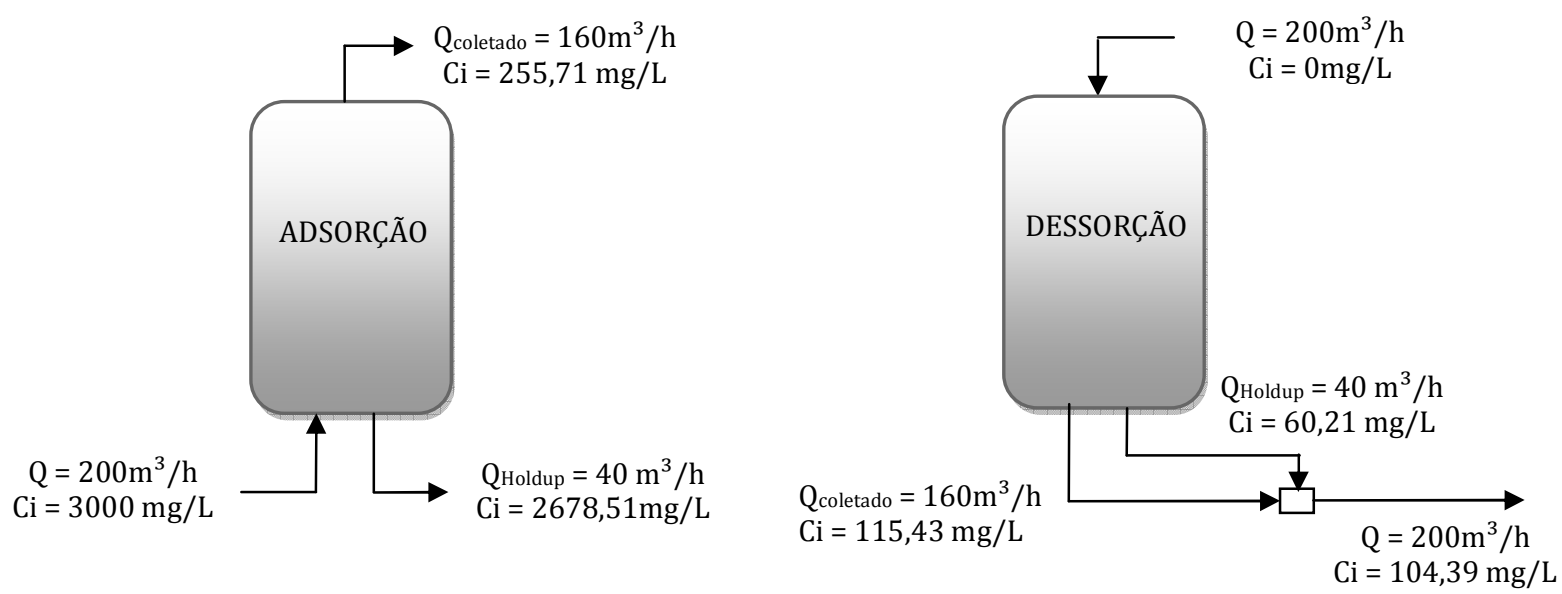

Figura 3 - Resultados obtidos na operação de uma coluna no estado estacionário 
Tabela 2 - Resultados da Simulação, conforme dados de entrada e parâmetros da Tabela 1.

\begin{tabular}{l|c}
\hline Vazão de Saída da Carga Tratada & $160 \mathrm{~m}^{3} / \mathrm{h}$ \\
\hline Conc. Média da Carga Tratada. & $255,71 \mathrm{mg} / \mathrm{L}$ \\
\hline Vazão do hold-up & $40 \mathrm{~m}^{3} / \mathrm{h}$ \\
\hline Conc. Média do hold-up & $2678,51 \mathrm{mg} / \mathrm{L}$ \\
\hline Vazão de Dessorvente & $200 \mathrm{~m}^{3} / \mathrm{h}$ \\
\hline $\begin{array}{l}\text { Conc. Média no Dessorvente Encaminhado } \\
\text { para a Unidade de Recuperação }\end{array}$ & $104,39 \mathrm{mg} / \mathrm{L}$ \\
\hline
\end{tabular}

\section{CONCLUSÕES}

Foi desenvolvido um algoritmo matemático capaz de descrever a operação contínua de uma unidade industrial de adsorção até atingir o estado estacionário cíclico, o qual poderá ser utilizado como ferramenta para equipes de projeto. Observa-se uma redução considerável nos teores de enxofre, mostrando que a adsorção por ser utilizada como tecnologia complementar ao processo tradicional de hidrodessulfurização.

\section{REFERÊNCIAS}

BASMADJIAN, D., The Little Adsorption Book: a practical guide for engineers and scientists, CRC Press, Boca Raton, Florida, 1997.

CERUTTI, M. L. M. N., Dessulfurização da Gasolina por Adsorção em Zeólitas "Y” Trocadas com Cobre, Tese - Centro Tecnológico, Universidade Federal de Santa Catarina, Santa Catarina, 2007.

FREITAS, T. C.; QUINTO, T. C.; SECCHI, A. R.; BISCAIA, E. C. An Efficient adjoint-free dynamic optimization methodology for batch processing using Pontryagin's formulation. In: BOGLE, I. D. L.; FAIRWEATHER, M. (Ed.) 22nd European Symposium on Computer Aided Process Engineering. Amsterdam: Elsevier, 2012.

RUTHVEN, D. M., Principles of Adsorption and Adsorption Processes. New York, John Wiley \& Sons, p. 433, 1984.

WHITEHURST, D. D., ISODA, T., MOCHIDA, I., Present State of the Art and Future Challenges in the Hydrodesulfurization of Polyaromatic Sulfur Compounds, Advan.Catal., v. 42, p345, 1998. 